PROCEEDINGS OF THE

AMERICAN MATHEMATICAL SOCIETY

Volume 132, Number 2, Pages 325-331

S 0002-9939(03)07154-5

Article electronically published on July 2, 2003

\title{
ON THE TANGENT SPACES OF CHOW GROUPS OF CERTAIN PROJECTIVE HYPERSURFACES
}

\author{
AVERY CHING \\ (Communicated by Michael Stillman)
}

\begin{abstract}
In this paper, the Chow groups of projective hypersurfaces are studied. We will prove that if the degree of the hypersurface is sufficiently high, its Chow group is "small" in the sense that its formal tangent space vanishes. Then, we will give an example in which the formal tangent space is infinite dimensional.
\end{abstract}

\section{INTRODUCTION}

In 7], Griffiths and Harris conjectured that for a generic threefold of degree $d \geq 6$, the Abel-Jacobi map from the cycles of codimension 2 homologous to zero to the intermediate Jacobian is zero. Later Green proved that [3] for projective hypersurfaces of dimension $2 m+1$ and degree $d \geq 2+4 / m$, the image of the AbelJacobi map on codimension $m+1$ cycles homologous to zero lands on the torsion of the intermediate Jacobian. The proof relied on the construction of the infinitesimal invariant of normal functions. Then he showed that these infinitesimal invariants vanish and proceeded with a monodromy argument.

In the case of cycles of other codimensions in a projective hypersurface, the intermediate Jacobian is zero and so the Abel-Jacobi map is not the right thing to look at. Instead, we look at the Beilinson filtration of their Chow groups, whose graded pieces are given by the formula

$$
G r^{m} C H^{p}(X)_{\mathbb{Q}}=\operatorname{Ext}_{\mathcal{M M}}^{m}\left(1, h^{2 p-m}(X)(p)\right),
$$

where $\mathcal{M M}$ is the conjectural category of mixed motive. For a projective hypersurface $X$ of dimension $n$, the only interesting cohomology is $H^{n}(X)$. Therefore, the only interesting graded piece of $C H^{p}(X)_{\mathbb{Q}}$ is the $(2 p-n)^{t h}$ piece. Only in the special cases $n=2 m+1$ and $p=m+1$ we will have $2 p-n=1$. Since the first graded piece of Chow groups under the conjectural filtration is identified with a subgroup of the intermediate Jacobian via the Abel-Jacobi map, the method of Abel-Jacobi maps is applied only to this special case.

For other cases, similar results should rely on the infinitesimal invariants of other graded pieces of Chow groups. The theory of the tangent spaces of Chow groups was developed first by Stienstra and Bloch, and later elaborated by Griffiths and Green [4. It serves as the analogue for infinitesimal invariants for normal functions.

Received by the editors May 20, 2001 and, in revised form, September 30, 2002.

2000 Mathematics Subject Classification. Primary 14C15; Secondary 19D45. 
In section 2 we will give a quick review of this theory, and then in section 3 we prove

Theorem 1. For a generic hypersurface $X$ of dimension $n$ in $\mathbb{P}^{n+1}$ with degree $d$, if

then

$$
(n-p) d \geq 2 p+1
$$

$$
\operatorname{TCH}^{p}(X)_{\mathbb{Q}}=0 .
$$

The tangent space of the rational $p^{\text {th }}$ Chow group is zero.

Finally, in section 4, we will give an example of explicit computation involving higher graded pieces of Chow groups.

This work was finished during the preparation of my Ph.D. thesis at UCLA. I thank M. Green, my thesis advisor, who educated me about Hodge theory and algebraic cycles from scratch. Special thanks go to P. Griffiths for some useful discussions.

\section{Tangent spaces of Chow groups}

Let $X$ be a complex variety of dimension $n$. A family of algebraic cycles of codimension $p$ over a parameter space $S$ is a codimension $p$ algebraic cycle $\mathcal{Z}$ of $X \times S$. We want to have an infinitesimal invariant of such a family.

Let $\mathcal{K}_{X}^{p}$ be the sheafification of the presheaf

$$
U \rightarrow K_{p}^{M}\left(\mathcal{O}_{X}(U)\right)
$$

where $K_{p}^{M}$ is the Milnor $K$-theory. There is a map of sheaves on $X$,

$$
\operatorname{dlog}: \mathcal{K}_{X}^{p} \rightarrow \Omega_{X / \mathbb{Q}}^{p}
$$

such that for any $f_{1}, \ldots, f_{p} \in \mathcal{O}_{X}(U)$,

$$
\operatorname{dlog}\left(\left\{f_{1}, \ldots, f_{p}\right\}\right)=\frac{1}{(2 \pi i)^{p}} \frac{d f_{1}}{f_{1}} \wedge \ldots \wedge \frac{d f_{p}}{f_{p}} .
$$

We also call the induced map in the $p^{\text {th }}$ cohomology dlog. Replace $X$ by the product $X \times S$ and consider the following compositions:

$$
\begin{aligned}
& C H^{p}(X \times S)_{\mathbb{Q}} \\
\rightarrow & H^{p}\left(X \times S, \mathcal{K}_{X \times S}^{p}\right) \\
\stackrel{\text { dlog }}{\rightarrow} & H^{p}\left(X \times S, \Omega_{X \times S / \mathbb{Q}}^{p}\right) \\
\rightarrow & H^{0}\left(S, \Omega_{S / \mathbb{C}}\right) \otimes H^{p-1}\left(X, \Omega_{X / \mathbb{Q}}^{p-1}\right),
\end{aligned}
$$

where the first map is obtained from Bloch's formula

$$
C H^{p}(X \times S) \stackrel{\cong}{\rightrightarrows} H^{p}\left(X \times S, \mathcal{K}_{X \times S}^{Q, p}\right),
$$

and where $\mathcal{K}_{X}^{Q, P}$ is similarly defined with Milnor's $K$-theory replaced by Quillen's $K$-theory. See [10] for details. Thanks to Van der Kallen's theory for the formal tangent space for the Milnor $K$ functor, we can define $H^{p}\left(X, \Omega_{X / \mathbb{Q}}^{p-1}\right)$ as the formal tangent space of the Chow group $C H^{p}(X)_{\mathbb{Q}}$, usually denoted by $T C H^{p}(X)_{\mathbb{Q}}$ (see [4] and [8]). Under these compositions the image of the rational equivalence class of $\mathcal{Z}$ is an element of

$$
H^{0}\left(S, \Omega_{S / \mathbb{C}}\right) \otimes T C H^{p}(X)_{\mathbb{Q}}
$$

which is the infinitesimal invariant of the family. 
There is a natural filtration on the sheaves $\Omega_{X / \mathbb{Q}}^{p-1}$, given by

$$
F i l t^{m} \Omega_{X / \mathbb{Q}}^{p-1}=i m\left(\Omega_{\mathbb{C} / \mathbb{Q}}^{m} \otimes \Omega_{X / \mathbb{Q}}^{p-1-m} \rightarrow \Omega_{X / \mathbb{Q}}^{p-1}\right) .
$$

The spectral sequence for this filtration degenerates at the $E_{2}$ term [2]. Thus, it induces a filtration on $H^{p}\left(\Omega_{X / \mathbb{Q}}^{p-1}\right)$ whose graded pieces are

$$
G r^{m} H^{p}\left(\Omega_{X / \mathbb{Q}}^{p-1}\right)=H^{m-1}\left(\Omega_{\mathbb{C} / \mathbb{Q}}^{\bullet} \otimes H^{p-1-\bullet, p-m+1+\bullet}(X), \nabla\right),
$$

where $\nabla$ is the arithmetic Gauss-Manin connection. It is related to the spread of $X$ and the geometric Gauss-Manin connection on it. We refer the reader to 4] for details.

In [4] it is conjectured that the above filtration of $T C H^{p}(X)_{\mathbb{Q}}$ is consistent with the conjectural filtration on Chow groups, i.e.,

$$
T G r^{m} C H^{p}(X)_{\mathbb{Q}}=H^{m-1}\left(\Omega_{\mathbb{C} / \mathbb{Q}}^{\bullet} \otimes H^{p-1-\bullet, p-m+1+\bullet}(X), \nabla\right) .
$$

From the construction of the infinitesimal invariant, it is clear that if a family has constant rational equivalence class, then its infinitesimal invariant is zero. However, we have to warn the reader that the converse is not true. For a counterexample, see [4]. Thus, the formal tangent space is not a tangent space in the usual sense.

\section{TANGent SPACES OF ChOW Groups of a PROJECtive hypersurface}

This section is devoted to the proof of Theorem 1

For a projective hypersurface $X$ in $\mathbb{P}^{n+1}$ defined by an equation $F \in H^{0}(\mathcal{O}(d))$, its cohomologies are particularly simple. First of all, the restriction of the Kähler class is a flat class because it is algebraic [1. So, the only possibly non-zero graded piece of $C H^{p}(X)_{\mathbb{Q}}$ is the $(2 p-n)^{t h}$ piece, which is the cohomology at the middle term of

$\Omega_{\mathbb{C} / \mathbb{Q}}^{2 p-n-2} \otimes H^{n-p+1, p-1}(X) \rightarrow \Omega_{\mathbb{C} / \mathbb{Q}}^{2 p-n-1} \otimes H^{n-p, p}(X) \rightarrow \Omega_{\mathbb{C} / \mathbb{Q}}^{2 p-n} \otimes H^{n-p-1, p+1}(X)$.

With the residue representation [6], the dual of the above sequence is

$\Omega_{\mathbb{C} / \mathbb{Q}}^{2 p-n *} \otimes R^{(n-p) d-n-2} \rightarrow \Omega_{\mathbb{C} / \mathbb{Q}}^{2 p-n-1 *} \otimes R^{(n-p+1) d-n-2} \rightarrow \Omega_{\mathbb{C} / \mathbb{Q}}^{2 p-n-2 *} \otimes R^{(n-p+2) d-n-2}$,

where $R^{k}$ is the Jacobi ring of the hypersurface at degree $k$. Now we describe the spread of $X$ and relate the arithmetic Gauss-Manin connection to the geometric Gauss-Manin of this spread.

Let $X$ be defined over a field $k=\mathbb{Q}\left(\alpha_{1}, \ldots, \alpha_{N}\right) /\left\langle h_{1}, \ldots, h_{M}\right\rangle=\mathbb{Q}(S)$, where $\alpha_{j}$ are transcendental numbers and $h_{j}$ are polynomial relations between them. $S$ is the subvariety of $\mathbb{A}^{N}$ defined by the equations $h_{j}$. Then $F(\alpha, z)$ can be written as a polynomial in $z_{i}$ and $\alpha_{j}$. Therefore, it gives a hypersurface $\mathcal{X}$ in $S \times \mathbb{P}^{5}$ defined by the equation $F\left(s_{1}, \ldots, s_{N}, z_{0}, \ldots, z_{n}\right)=0$. The projection $\mathcal{X} \rightarrow S$ (we may need to restrict to a Zariski open subset of $S$ and pass to an étale covering) is a family of projective hypersurfaces over $S$ in which $X$ is the fiber at $s_{0}$, say. Since $d s_{j}$ and the $d \alpha_{j}$ satisfy the same relations, we have an imbedding

$$
\begin{aligned}
\left.\Omega_{S}^{1}\right|_{s_{0}} & \stackrel{\iota}{ } \Omega_{\mathbb{C} / \mathbb{Q}}^{1}, \\
d s_{j} & \mapsto d \alpha_{j} .
\end{aligned}
$$

By [2], the arithmetic Gauss-Manin connection equals the composition of $\iota$ and the geometric Gauss-Manin connection at the point $s_{0}$. The dual of $\iota$ is a map

$$
\Omega_{\mathbb{C} / \mathbb{Q}}^{1 *} \rightarrow T_{s_{0}} S \hookrightarrow R^{d} .
$$


If $\alpha \in \mathbb{C}$, then $\frac{\partial}{\partial \alpha} \in \Omega_{\mathbb{C} / \mathbb{Q}}^{1 *}$ is mapped to $\frac{\partial F}{\partial \alpha} \in R^{d}$. Let $W$ be the subspace of $R^{d}$ spanned by all these polynomial classes as $\alpha$ runs through all complex numbers. The sequence giving the tangent space of Chow groups becomes

$$
\begin{aligned}
\bigwedge^{2 p-n} W \otimes R^{(n-p) d-n-2} & \rightarrow \bigwedge^{2 p-n-1} W \otimes R^{(n-p+1) d-n-2} \\
& \rightarrow \bigwedge^{2 p-n-2} W \otimes R^{(n-p+2) d-n-2},
\end{aligned}
$$

where the differentials are the geometric Gauss-Manin on the spread, which is well known to be polynomial multiplication. Therefore, this sequence is the standard Kozul complex described in [5]. It is exact at the middle term if

$$
(n-p+1) d-n-2 \geq(2 p-n-1)+d+\operatorname{codim}(W)
$$

[5], i.e.,

$$
(n-p) d \geq 2 p+1+\operatorname{codim}(W) .
$$

In the generic case in which all coefficients of $F$ are algebraically independent, $\operatorname{codim}(W)=0$. We see that under the criterion

$$
(n-p) d \geq 2 p+1,
$$

we have the vanishing of the cohomology

$$
H^{2 p-n-1}\left(\Omega_{\mathbb{C} / \mathbb{Q}}^{\bullet} \otimes H^{p-1-\bullet, n-p+1+\bullet}(X), \nabla\right) .
$$

Thus, Theorem 1 is proved. Note that we can also apply residue representation to cohomologies of complete intersections, and there is a similar criterion for the vanishing of the corresponding Kozul complex [9]. Going through the same procedure as above yields

Theorem 2. If $X$ is a general complete intersection of dimension $n$ in $\mathbb{P}^{n+r}$ of multi-degree $\left(d_{1}, \ldots, d_{r}\right)$, and if

$$
(n-p) \min _{j}\left\{d_{j}\right\} \geq 2 p+r,
$$

then $T C H^{p}(X)_{\mathbb{Q}}=0$.

\section{QUintic FOURFOLDS}

In the case when $X$ is a quintic fourfold in $\mathbb{P}^{5}$, defined by $F$, the result in the previous section shows that $T C H^{3}(X)_{\mathbb{Q}}$ does not necessarily vanish. In this section, we give the infinitesimal invariant of a family of cycles on $X$. The family $\Sigma$ we consider is the two parameters family of lines on $X$. The difference between a line $L$ and a fixed one $L_{0}$ is homologous to zero. Since $H^{5}(X)=0$, the first graded piece of $C^{3}(X)_{\mathbb{Q}}$ is also zero. So, the infinitesimal invariant of the family is a class in

$$
T^{*} \Sigma \otimes H^{1}\left(\Omega_{\mathbb{C} / \mathbb{Q}}^{\bullet} \otimes H^{2-\bullet, 2+\bullet}(X), \nabla\right) .
$$

Suppose that $X$ is defined over $\mathbb{Q}$ for simplicity. Then the arithmetic Gauss-Manin connection is zero and the above space simplifies to

$$
T^{*} \Sigma \otimes \Omega_{\mathbb{C} / \mathbb{Q}}^{1} \otimes H^{1,3}(X) .
$$

To specify the infinitesimal invariant is to give a tri-linear map

$$
T \Sigma \otimes \Omega_{\mathbb{C} / \mathbb{Q}}^{1 *} \otimes H^{3,1}(X) \rightarrow \mathbb{C}
$$


given by cup product and contraction, just like the ordinary derivative of period maps. Now, by general deformation theory

$$
T_{L} \Sigma=H^{0}\left(N_{L / X}\right)
$$

and there is a natural map

$$
\Omega_{\mathbb{C} / \mathbb{Q}}^{1, *} \rightarrow H^{0}\left(N_{L / X}\right)
$$

constructed as follows. Let $\alpha \in \mathbb{C}$, and $L$ is defined by the linear equations $l_{j} \in$ $H^{0}\left(\mathcal{O}_{\mathbb{P}^{5}}(1)\right), j \in\{0, \ldots, 3\}$. Then we can write $F$ as

$$
F=\sum_{j} G_{j} l_{j}
$$

for some $G_{j} \in H^{0}\left(\mathcal{O}_{\mathbb{P}^{5}}(4)\right)$. Differentiating with respect to $\alpha$ yields

$$
0=\sum_{j} \frac{\partial G_{j}}{\partial \alpha} l_{j}+\sum_{j} G_{j} \frac{\partial l_{j}}{\partial \alpha}
$$

Therefore,

$$
\left.\sum_{j} G_{j} \frac{\partial l_{j}}{\partial \alpha}\right|_{L}=0
$$

The four linear forms $\left(\left.\frac{\partial l_{0}}{\partial \alpha}\right|_{L}, \ldots,\left.\frac{\partial l_{3}}{\partial \alpha}\right|_{L}\right)$ can be considered as an element in $H^{0}\left(N_{L / \mathbb{P}^{5}}\right)$ which lies in the kernel of

$$
H^{0}\left(N_{L / \mathbb{P}^{5}}\right) \rightarrow H^{0}\left(\left.N_{X / \mathbb{P}^{5}}\right|_{L}\right) .
$$

From the exact sequence

$$
\left.0 \rightarrow N_{L / X} \rightarrow N_{L / \mathbb{P}^{5}} \rightarrow N_{X / \mathbb{P}^{5}}\right|_{L} \rightarrow 0,
$$

we see that it comes from a unique element in $N_{L / X}$. It is routine to verify that this element in $N_{L / X}$ does not depend on the choice of $l_{j}$ and thus gives a well defined map

$$
\Omega_{\mathbb{C} / \mathbb{Q}}^{1 *} \rightarrow H^{0}\left(N_{L / X}\right)
$$

Since $X$ is defined over $\mathbb{Q}$, the spread of the family is the spread of $L$. The tangent space of this spread is isomorphically mapped to $H^{0}\left(N_{L / X}\right)$ via the above map. So, the infinitesimal invariant is given by

$$
\begin{aligned}
& T^{*} \Sigma \otimes \Omega_{\mathbb{C} / \mathbb{Q}}^{1 *} \otimes H^{3,1}(X) \\
\rightarrow & \wedge^{2} H^{0}\left(N_{L / X}\right) \otimes H^{3,1}(X) \\
\rightarrow & \mathbb{C},
\end{aligned}
$$

where the last map is given by cup product and contraction. We apply the residue representation to identify $H^{3,1}(X)$ with $H^{0}\left(\mathcal{O}_{\mathbb{P}^{5}}(4)\right)$. There is a simple description of the residue in this simple case. Let $e \in H^{1}\left(T_{X}(-5)\right)$ be the extension class of

$$
0 \rightarrow N_{X / \mathbb{P}^{5}}^{*} \rightarrow \Omega_{\mathbb{P}^{5}}^{1} \rightarrow \Omega_{X}^{1} \rightarrow 0 .
$$

By the adjunction formula $N_{X / \mathbb{P}^{5}}=\mathcal{O}(5)$, the exact sequence

$$
\left.0 \rightarrow \Omega_{X}^{3} \rightarrow \Omega_{\mathbb{P}^{5}}^{4}\right|_{X}(5) \rightarrow \Omega_{X}^{4}(5) \rightarrow 0
$$

has an extension class also given by $e$, i.e., the connecting homomorphism

$$
H^{0}(\mathcal{O}(4)) \rightarrow H^{0}\left(K_{X}(5)\right) \rightarrow H^{1}\left(\Omega_{X}^{3}\right),
$$


which is the residue representation of $H^{3,1}(X)$, is given by multiplication with $e$. Now, consider the exact sequence

$$
\left.0 \rightarrow N_{L / X}(-1) \rightarrow N_{L / \mathbb{P}^{5}}(-1) \rightarrow N_{X / \mathbb{P}^{5}}\right|_{L}(-1) \rightarrow 0 .
$$

The connecting homomorphism

$$
H^{0}\left(\mathcal{O}_{L}(4)\right) \rightarrow H^{1}\left(N_{L / X}(-1)\right)
$$

factors through

$$
H^{0}\left(\mathcal{O}_{L}(4)\right) \rightarrow H^{1}\left(\left.T_{X}\right|_{L}(-1)\right) \rightarrow H^{1}\left(N_{L / X}(-1)\right),
$$

where the first map is to multiply with $e$ and then contract, and the second map is the natural projection. But in $H^{1}\left(T_{L}(-1)\right)=0$, the second map is an isomorphism. Therefore, the residue representation can be realized as

$$
H^{0}\left(\mathcal{O}_{L}(4)\right) \rightarrow H^{1}\left(N_{L / X}(-1)\right) .
$$

Thus our infinitesimal invariant is the compositions

$$
\begin{aligned}
& H^{0}\left(N_{L / X}\right) \otimes \Omega_{\mathbb{C}}^{1 * \mathbb{Q}} \otimes H^{3,1}(X) \\
\rightarrow & H^{0}\left(N_{L / X}\right) \otimes H^{0}\left(N_{L / X}\right) \otimes H^{0}(\mathcal{O}(4)) \\
\rightarrow & \bigwedge^{2} H^{0}\left(N_{L / X}\right) \otimes H^{1}\left(N_{L / X}(-1)\right) \\
\rightarrow & H^{1}\left(\bigwedge^{3} N_{L / X}(-1)\right) .
\end{aligned}
$$

Finally, we note that $N_{L / X}=\mathcal{O}(-1) \oplus \mathcal{O} \oplus \mathcal{O}$. Therefore,

$$
\bigwedge^{3} N_{L / X}(-1)=\mathcal{O}(-2)=K_{L}
$$

and

$$
H^{1}\left(\bigwedge^{3} N_{L / X}(-1)\right)=H^{1}\left(K_{L}\right)=\mathbb{C} .
$$

Summarizing everything in this section, we have

Theorem 3. For a quintic fourfold $X$ defined over $\mathbb{Q}$, let $\Sigma$ be the two-dimensional parameter space of lines on $X$. Then, the family has an infinitesimal invariant

$$
T \Sigma \rightarrow \Omega_{\mathbb{C} / \mathbb{Q}}^{1} \otimes H^{1,3}(X)
$$

induced by

$$
\begin{aligned}
& T \Sigma \otimes \Omega_{\mathbb{C} / \mathbb{Q}}^{1 *} \otimes H^{3,1}(X) \\
\rightarrow & H^{0}\left(N_{L / X}\right) \otimes H^{0}\left(N_{L / X}\right) \otimes H^{0}(\mathcal{O}(4)) \\
\rightarrow & \bigwedge^{2} H^{0}\left(N_{L / X}\right) \otimes H^{1}\left(N_{L / X}(-1)\right) \\
\rightarrow & H^{1}\left(\bigwedge^{3} N_{L / X}(-1)\right) \\
\rightarrow & H^{1}\left(K_{L}\right) \\
\rightarrow & \mathbb{C} .
\end{aligned}
$$

This map is nonzero whenever $\Omega_{\mathbb{C} / \mathbb{Q}}^{1 *} \rightarrow H^{0}\left(H_{L / X}\right)$ is nonzero, which happens in the generic case. So, we have

Corollary 1. Two general lines on a general quintic fourfold defined over $\mathbb{Q}$ are not rationally equivalent. 


\section{REFERENCES}

[1] Deligne, P., Milne, J. S., Ogus, A., and Shih, K., Hodge cycles, motives and Shimura varieties, Lecture Notes in Math. 900, Springer-Verlag, Berlin, 1982. MR 84m:14046

[2] Esnault, H. and Paranjape, K., Remarks on absolute de Rham and absolute Hodge cycles, C. R. Acad. Sci. Paris, no. 319, 67-72 (1994) MR 95d:14008

[3] Green, M. L., Griffiths' infinitesimal invariant and the Abel-Jacobi map, J. Differential Geom. no. 29, 545-555 (1989) MR 90c:14006

[4] Green, M. L., Banff lecture notes, 1998

[5] Green, M. L., Infinitesimal methods in Hodge theory, Lecture Notes in Math., no. 1594, Springer-Verlag, Berlin, 1994. MR 96m:14012

[6] Griffiths, P., On the periods of certain rational integrals, I, II, Annals of Math., no. 90, 460-541 (1969) MR 41:5357

[7] Griffiths, P. and Harris, J., On the Noether-Lefschetz theorem and some remarks on codimension two cycles, Math. Ann., no. 271, 31-51 (1985) MR 87a:14030

[8] van der Kallen, W., Generators and relations in algebraic K-theory, Proc. Internat. Congress Math., Helsinki 1978, 305-310 (1978) MR 81g:18017

[9] Konno, K., On the variational Torelli problem for complete intersections, Compositio Math. 78, 271-296 (1991) MR 92f:14009

[10] Quillen, D., Higher algebraic K-theory, I, Lecture Notes in Math. 341, Springer-Verlag, 1973. MR 49:2895

Department of Mathematics, Johns Hopkins University, Baltimore, Maryland 212182686

E-mail address: aching@math.jhu.edu 